\title{
Theory of non-Fermi liquid near a diagonal electronic nematic state on a square lattice
}

\author{
Ying-Jer Kao* \\ Department of Physics and Center for Theoretical Sciences, National Taiwan University, Taipei 106, Taiwan \\ Hae-Young $\mathrm{Kee}^{\dagger}$ \\ Department of Physics, University of Toronto, Toronto, Ontario, Canada M5S 1A7 \\ (Received 5 February 2007; revised manuscript received 9 May 2007; published 13 July 2007)
}

\begin{abstract}
We study the effects of Fermi-surface fluctuations on the single-particle lifetime near the diagonal electronic nematic phase on a two-dimensional square lattice. It has been shown that there exists a quantum critical point between the diagonal nematic and isotropic phases [H. Doh et al., Phys. Rev. B 73, 125117 (2006)]. We study the longitudinal fluctuations of the order parameter near the critical point, where the singular forward scattering leads to a non-Fermi-liquid behavior over the whole Fermi surface except along the $k_{x}$ and $k_{y}$ directions. We will also discuss the temperature and chemical potential dependences of the single-particle decay rate.
\end{abstract}

DOI: $10.1103 /$ PhysRevB.76.045106

PACS number(s): 71.10.Hf, 71.27.+a

\section{INTRODUCTION}

In strongly correlated electronic materials, electrons can organize themselves into a pattern with periodicity differing from that of the underlying lattice along a particular direction. The phase with such a nontrivial charge ordering breaks a discrete translational symmetry of the underlying lattice along one direction and has been named as a stripe. Stripe physics has been of great interest to both theoretical and experimental condensed-matter physicists, especially because its quantum fluctuations may play an important role in understanding the mechanism of high-temperature superconductivity. ${ }^{2-4}$ Clear evidence of such a novel paired stripe order was reported by Mori et al. in electrondiffraction experiment on $(\mathrm{La}, \mathrm{Ca}) \mathrm{MnO}_{3}{ }^{5}$

It has been proposed that phases with fluctuating stripes can be generic ground states of a doped Mott insulator introduced by hole doping. ${ }^{6,7}$ These intermediate forms of matter have been dubbed as electronic smectic and nematic phases. While the smectic breaks a translational symmetry, the electronic nematic phase breaks a discrete rotational symmetry of the underlying lattice and can be viewed as fluctuating stripes. The electronic nematic phase has been referred to as the Pomeranchuk instability of a Fermi liquid ${ }^{8}$ and has been studied in theoretical models such as the $t-J$, (extended) Hubbard, and Fermi-liquid models. ${ }^{9-12}$ The electronic nematic phase has been suggested as being responsible for novel phenomena observed in strongly correlated systems. These phenomena include, for example, anisotropic resistivities in high Landau levels of quantum Hall systems,${ }^{13}$ anisotropic scattering patterns observed in one of the high-temperature superconductors, ${ }^{14,15}$ and the metamagnetic transition and anisotropic transport observed in $\mathrm{Sr}_{3} \mathrm{Ru}_{2} \mathrm{O}_{7}{ }^{16-18}$

In addition to the anisotropic nature of the electronic nematic phase, it has been shown that the gapless Goldstone mode of the nematic phase in a continuum model leads to non-Fermi-liquid behavior in the single-particle scattering rate on the Fermi surface except along the symmetric directions. ${ }^{19}$ However, using the same model of quadrupole density interaction on the square lattice, it is shown that the putative continuous transition is pre-empted by a first-order transition due to a Van Hove singularity. The regime of the nematic phase diminishes exponentially as one approaches the continuum limit of small densities with an approximately circular Fermi surface. ${ }^{20,21}$ Therefore, there are no critical Fermi-surface fluctuations for non-Fermi-liquid behavior in realistic systems with an underlying lattice; both transverse (phase) and longitudinal (amplitude) modes are gapped. On the other hand, it was later found that an additional uniform interaction in the forward scattering channel suppresses the first-order transition. ${ }^{22}$ This restores the non-Fermi-liquid behavior at the Fermi surface, except along the zone diagonal direction, via a coupling to the fluctuations of the order parameter at the quantum critical point. ${ }^{23}$

In this paper, we present a theory of the non-Fermi liquid near the diagonal nematic phase. It is worthwhile to note that there are two distinct nematic states in the square lattice. ${ }^{1}$ Previous studies provide an understanding of the phenomena related to Fermi-surface distortions along the directions parallel to the $k_{x}$ or $k_{y}$ axes of the Brillouin zone. The diagonal nematic phase can be characterized by a Fermi-surface distortion along the diagonal direction of the Brillouin zone. It is shown that the transition between the diagonal nematic and isotropic phases is of second order, because it does not involve a splitting of the Van Hove singularity in the density of states. ${ }^{1}$ We find non-Fermi-liquid behavior in the singleparticle lifetime at the Fermi surface through the coupling to the longitudinal mode of the diagonal nematic order, except along the zone parallel directions $\left(k_{x}\right.$ and $k_{y}$ axes of the Brillouin zone). Our numerical results for the self-energy show the expected exponent of $2 / 3$ in frequency, similar to the parallel nematic phase ${ }^{23}$ and the continuum model. ${ }^{19}$ However, the physical origin of the non-Fermi-liquid behavior is different for these three cases.

\section{MODEL}

The model Hamiltonian for the parallel and the diagonal nematic phases on a square lattice is written as follows: 


$$
\begin{aligned}
H= & \sum_{\mathbf{k} \sigma} \varepsilon_{\mathbf{k}} c_{\mathbf{k} \sigma}^{\dagger} c_{\mathbf{k} \sigma}-\sum_{\mathbf{k} \mathbf{k}^{\prime} \mathbf{q} \sigma \sigma^{\prime}}\left[F_{2}(\mathbf{q}) \zeta_{1}(\mathbf{k}) \zeta_{1}\left(\mathbf{k}^{\prime}\right)\right. \\
& \left.+G_{2}(\mathbf{q}) \zeta_{2}(\mathbf{k}) \zeta_{2}\left(\mathbf{k}^{\prime}\right)\right] c_{\mathbf{k}+(\mathbf{q} / 2) \sigma^{\dagger}} c_{\mathbf{k}^{\prime}-(\mathbf{q} / 2) \sigma^{\prime}}^{\dagger} c_{\mathbf{k}^{\prime}+\mathbf{q}_{/}^{2} \sigma^{\prime}} c_{\mathbf{k}-(\mathbf{q} / 2) \sigma},
\end{aligned}
$$

where $\zeta_{1}(\mathbf{k})=\cos k_{x}-\cos k_{y}$ and $\zeta_{2}(\mathbf{k})=2 \sin k_{x} \sin k_{y}$ are the form factors originating from the quadrupole density-density interaction, ${ }^{19}$ and $\varepsilon_{\mathbf{k}}$ is the tight-binding dispersion $\varepsilon_{\mathbf{k}}=$ $-2 t\left(\cos k_{x}+\cos k_{y}\right)-\mu$. We assume short-range quadrupolar interactions of $F_{2}(\mathbf{q})$ and $G_{2}(\mathbf{q})$ with the form of $F_{2} /(1$ $\left.+\kappa q^{2}\right)$ and $G_{2} /\left(1+\kappa q^{2}\right)$, respectively.

The order parameters associated with the parallel $\left(\Delta_{P}\right)$ and diagonal $\left(\Delta_{D}\right)$ nematic phases are given by

$$
\begin{aligned}
& \Delta_{P}=F_{2} \sum_{\mathbf{k}}\left(\cos k_{x}-\cos k_{y}\right)\left\langle c_{\mathbf{k}}^{\dagger} c_{\mathbf{k}}\right\rangle, \\
& \Delta_{D}=G_{2} \sum_{\mathbf{k}}\left(2 \sin k_{x} \sin k_{y}\right)\left\langle c_{\mathbf{k}}^{\dagger} c_{\mathbf{k}}\right\rangle .
\end{aligned}
$$

Solving the above mean-field equation, Eq. (2), one can obtain the behavior of the nematic order parameters as one varies the ratio of $F_{2} / G_{2}$. The transition to the diagonal nematic ordered state from the isotropic liquid phase occurs above a critical value of the interaction $G_{2}$, and it is of second order as a function of the chemical potential. The competition between the diagonal and the parallel nematics leads to the suppression of both phases, while they coexist in a finite window of chemical potential. ${ }^{1}$ Longer-range hopping, such as $t^{\prime}$, is ignored in this study, since the qualitative features are not affected by a finite $t^{\prime},{ }^{1,20}$

To study the behavior of the non-Fermi liquid near the quantum critical point (QCP), we set our parameter as follows: $F_{2} N_{0}=0.1$ and $G_{2} N_{0}=0.196$, where $N_{0}=1 /\left(2 t \pi^{2}\right)$ is the density of states at the Fermi surface. With these parameters, the QCP occurs at $\mu_{c} /(2 t)=-0.19$. While the parallel nematic also occurs at smaller value of $\mu$ within the above parameter space, below we will present the results near the $\mu_{c}$, since the non-Fermi-liquid behavior is realized only near the QCP associated with the diagonal nematic phase. We first consider the dynamical effective interaction between quasiparticles.

\section{SUSCEPTIBILITY IN RANDOM-PHASE APPROXIMATION}

The effective interaction mediated by the collective modes at the random-phase-approximation level is given by

$$
\chi_{N}(\mathbf{q}, \nu)=\frac{G_{2}(\mathbf{q})}{1-G_{2}(\mathbf{q}) \Pi_{N}^{0}(\mathbf{q}, \nu)},
$$

and the bare dynamical polarizability of the diagonal nematics is given by
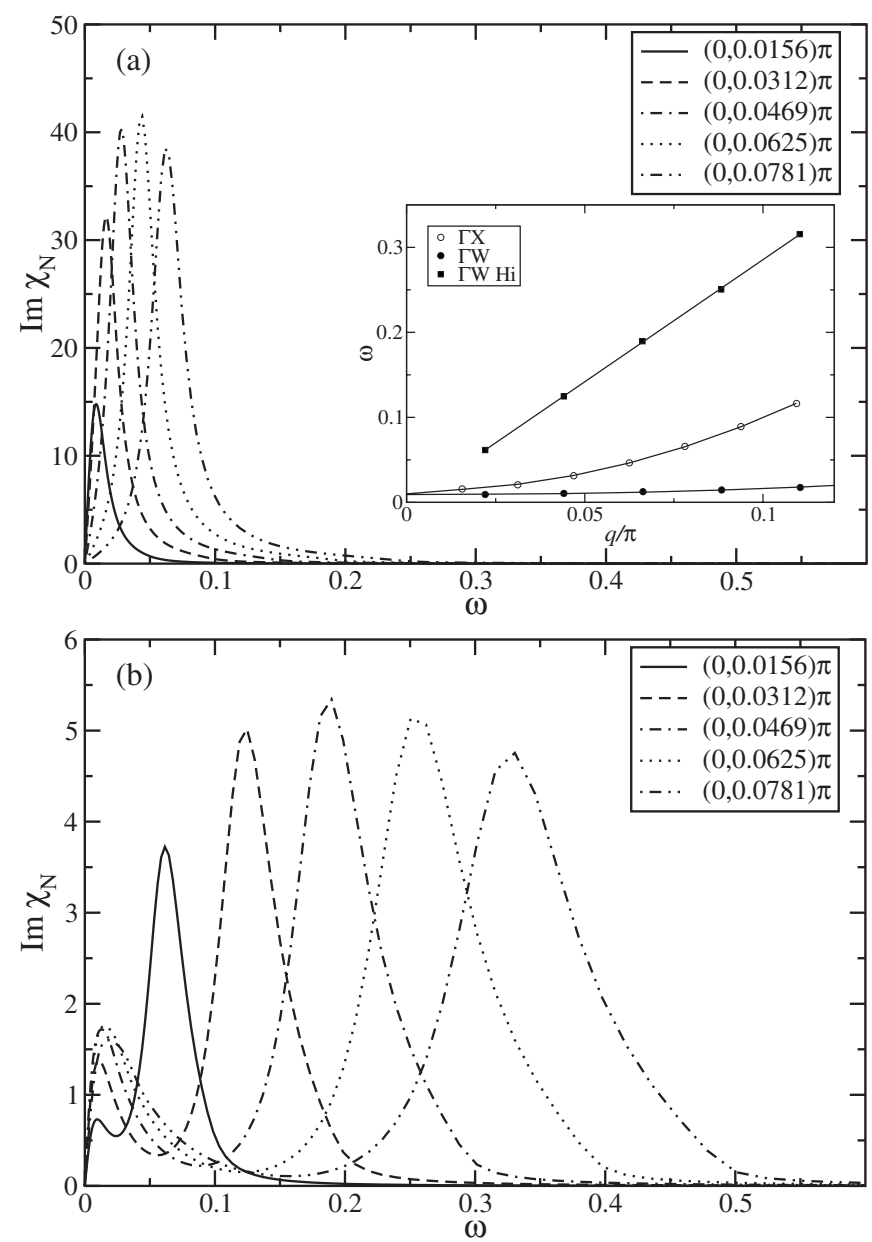

FIG. 1. The spectral function of the effective interaction, $\operatorname{Im} \chi_{N}$, as a function of $\omega$ slightly away from the QCP $(\mu=-0.22)$ for several choices of $\mathbf{q}$ along the (a) zone parallel $(0, \pi)$ and (b) zone diagonal $(\pi, \pi)$ directions. There is a sharp propagating mode along the $(0, \pi)$ direction, and the mode is diffusive along the zone diagonals. There is also a sound-mode-like mode at higher energy. Inset: dispersion of the collective modes along the $\Gamma W(\pi, \pi)$ (solid symbols) and $\Gamma X(0, \pi)$ (open symbols) directions. The lower-energy modes show a $q^{2}$ dependence in both directions except at (sufficiently) low $q$. The high-energy mode is soundlike with the dispersion $\omega=v q$.

$$
\Pi_{N}^{0}(\mathbf{q}, \nu)=-\int \frac{d^{2} p}{(2 \pi)^{2}} \frac{f\left(\varepsilon_{\mathbf{p}+\mathbf{q} / 2}\right)-f\left(\varepsilon_{\mathbf{p}-\mathbf{q} / 2}\right)}{\nu+i \eta-\left(\varepsilon_{\mathbf{p}+\mathbf{q} / 2}-\varepsilon_{\mathbf{p}-\mathbf{q} / 2}\right)} \zeta_{2}(\mathbf{p})^{2},
$$

where $f(\epsilon)$ is the Fermi function. Near the nematic transition, the denominator in Eq. (3) becomes very small for $\mathbf{q} \rightarrow 0$ and $\nu \rightarrow 0$, if $\nu$ vanishes faster than $\mathbf{q}$. This results in a singular behavior of the effective interaction in the forward scattering limit and, eventually, non-Fermi-liquid behavior.

We carried out numerical integrations of the above equation. In Fig. 1, the imaginary part of the effective interaction for the diagonal nematic is shown for small values of $q$ for $\mu / 2 t=-0.22$ slightly above the QCP in the symmetric phase. The diagonal nematic fluctuations have a propagating mode 
along the zone parallel directions and the mode becomes diffusive along the zone diagonals $[(\operatorname{Im} \omega) / \omega$ is peaked at $\omega=0]$. There also exists a soundlike mode at higher energy along the zone diagonals. These can be contrasted with the nematic Goldstone modes in the continuum case. ${ }^{19,24}$

In the inset of Fig. 1, we plot the dispersion of these modes. For the highly damped mode along the diagonal direction, the dispersion is determined by the peak position of the imaginary part of the effective interaction, while the mode dispersion along the zone parallel directions is given by the solution of $1-G_{2}(\mathbf{q}) \Pi_{0}(\mathbf{q}, \omega(\mathbf{q}))=0$. The lowerenergy modes show a $q^{2}$ dependence in both directions, except at very low $q$ values with a finite but small gap of $\delta / 2 t=0.01$. This mode becomes gapless only at the QCP and the effective interaction becomes singular. The mode acquires a gap away from the QCP due to the discrete nature of the point-group symmetries of the underlying lattice. However, the numerical integration becomes nontrivial when $q$ $\rightarrow 0$ when $\nu$ vanishes faster than $q$.

\section{QUASIPARTICLE SELF-ENERGY}

To capture the singular behavior of the effective interaction, we expand the $\Pi_{N}^{0}$ in the small $q$ and small $\omega / q$ limit as follows:

$$
\Pi_{N}^{0} \approx a+b q^{2}+i c \frac{\omega}{q}+O\left(\frac{\omega^{2}}{q^{2}}\right),
$$

where the coefficients $a, b$, and $c$ can be determined numerically and depend on the underlying band structure ${ }^{23,25}$ and temperature. The closeness to the QCP can thus be quantified by the gap, $\delta=1-G_{2}(0) a$. To understand how the decay rate for single-particle excitations is modified by the collective fluctuations, we calculate the imaginary part of the selfenergy. At the one-loop level, the imaginary part of the selfenergy at real frequencies is given by

$$
\begin{aligned}
\operatorname{Im} \Sigma(\mathbf{k}, \omega)= & -\int \frac{d \nu}{2 \pi} \int \frac{d^{2} q}{(2 \pi)^{2}} \zeta_{2}(\mathbf{k}) \zeta_{2}\left(\mathbf{k}+\frac{\mathbf{q}}{2}\right)[b(\nu) \\
& +f(\nu+\omega)] \operatorname{Im} \chi_{N}(\mathbf{q}, \nu) \operatorname{Im} G(\mathbf{k}+\mathbf{q}, \omega+\nu) .
\end{aligned}
$$

In the lowest-order approximation, we use the noninteracting Green's function and $\operatorname{Im} G_{0}(\mathbf{k}, \omega)=-\pi \delta\left(\omega-\epsilon_{\mathbf{k}}\right)$ in Eq. (6). Figure 2 shows the imaginary parts of the self-energies in the symmetric phase $(\mu / 2 t=-0.3,-0.25)$, at the QCP $(\mu / 2 t$ $=-0.19)$, and inside the diagonal nematic phase $(\mu / 2 t$ $=-0.18,-0.12)$ at the Fermi surface along the $(\pi, \pi)$ direction in the low- $T$ limit. Near the QCP, the self-energy shows a clear deviation from Fermi-liquid behavior with the asymptotic form $\operatorname{Im} \Sigma \sim|\omega|^{\alpha}, \alpha \leqslant 1$. The scattering rate is largest at the QCP, $\mu / 2 t=-0.19$, and the self-energy shows a strong non-Fermi-liquid behavior $\alpha \sim 2 / 3$ at low frequencies. ${ }^{23,26}$ This indicates that the quasiparticle excitations are not well defined approaching the Fermi surface, which rules out a Fermi-liquid description. This non-Fermiliquid behavior exists at all parts of the Fermi surface except at four points along the $k_{x}$ and $k_{y}$ axes, where the quasipar-

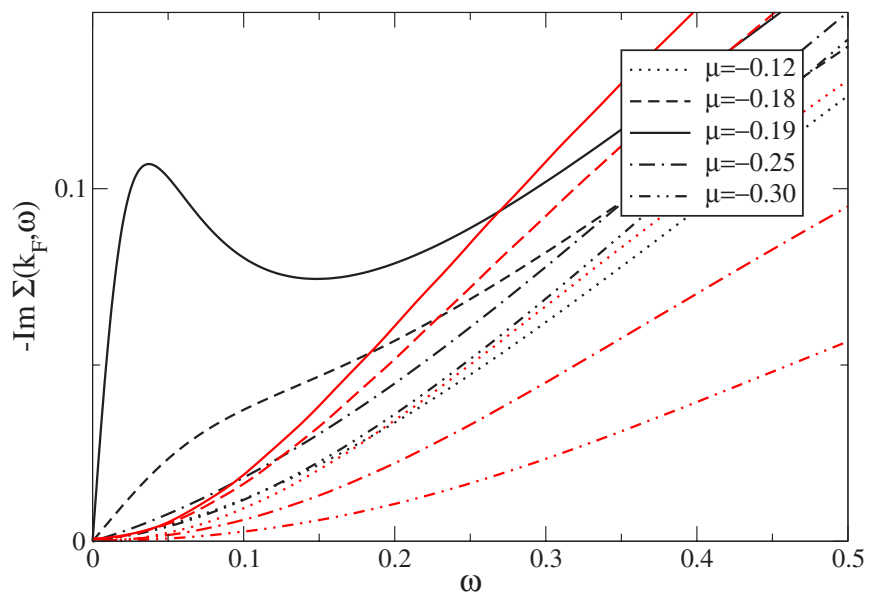

FIG. 2. (Color online) Calculated frequency dependence of the quasiparticle self-energy at the Fermi surface along the $(\pi, \pi)$ direction at different chemical potentials at $T=0 . \mu=-0.3,-0.25$ correspond to the symmetric phase, and $\mu=-0.18,-0.12$ are inside the diagonal nematic phase. $\mu=-0.19$ is the QCP and shows nonFermi-liquid behavior at $\omega \rightarrow 0$. For $\mu=-0.18$, a crossover from $\alpha$ $\sim 2 / 3$ to $\alpha \sim 1$ occurs at low frequency, indicating a small gap in the collective mode spectrum. Away from the QCP, we observe a crossover from a non-Fermi-liquid to a Fermi-liquid behavior. The red curves are the contributions from non-singular parts of the susceptibility. All quantities are in units of $2 t$.

ticle is well defined. Away from the QCP such as $\mu=-0.18$, the exponent crosses over to $\alpha \sim 1$, indicating a small but finite gap in the diagonal nematic collective mode at low frequencies. At other dopings, Fermi-liquid behavior is restored at low frequencies. We also compute the contribution from the regular part of $\Pi_{N}^{0}$ to the self-energy, and we find that the amplitude of the nonsingular part is always smaller than that of the singular part. This is due to the smaller density of states at the Fermi surface along the nodal directions. This should be contrasted with the parallel nematic order, where the density of states at the Fermi surface is large due to the closeness of the Van Hove singularity near $(\pi, 0)$.

In Fig. 3, we show the imaginary part of the self-energy at the Fermi surface in the nodal direction with $\mu / 2 t=-0.19$ at different temperatures. This corresponds to the quantum critical regime above the QCP. ${ }^{27,28}$ Finite temperature effects enter through the finite temperature Bose and Fermi functions, as well as the temperature dependence of the expansion coefficients in $\Pi_{N}^{0}$. The self-energy shows non-Fermiliquid behavior at small $\omega$ for all temperatures. In the quantum critical regime at $T>0$, the self-energy consists of contributions from both the classical and the quantum fluctuations. The quantum part of the self-energy obeys $(\omega / T)$ scaling in the quantum critical regime, while the classical part dominates at $\omega=0$ and scales as $\operatorname{Im} \Sigma\left(\mathbf{k}_{F}, 0\right) \propto T \xi(T)$ $\propto \sqrt{T / \ln T},{ }^{23,27}$ We observe this scaling of the classical fluctuations in $\operatorname{Im} \Sigma\left(\mathbf{k}_{F}, 0\right)$; however, our numerical precision cannot separate these two contributions.

\section{CONCLUSION}

A consequence of the nematic ordering is the distortion of the underlying Fermi surface. It has been proposed that the 
Fermi-surface distortion and critical Fermi-surface fluctuations play an important role in strongly correlated electron systems. Recent experiments in $\mathrm{Sr}_{3} \mathrm{Ru}_{2} \mathrm{O}_{7}$ near the metamagnetic QCP suggest that the formation of a spin-dependent nematic phase can explain the experimental observation of strong anisotropy in the magnetoresistivities. ${ }^{16,29}$ It has also been suggested that the in-plane anisotropy of the spin dynamics in detwinned $\mathrm{YBa}_{2} \mathrm{Cu}_{3} \mathrm{O}_{7-\delta}$ indicates the possible existence of a two-dimensional anisotropic liquid crystalline phase in the high-temperature cuprates. ${ }^{14,15,30}$ Close to the Pomeranchuk instability of the electronic system, the dynamics of the lattice distortion can be dramatically amplified. It has been argued that the electron-phonon interaction indeed plays an important role in the high- $T_{c}$ superconductors. ${ }^{31}$ The anisotropic form factors of the $B_{1 g}$ buckling phonons have the form of $\left(\cos k_{x}-\cos k_{y}\right){ }^{31}$ Therefore, it is interesting to note that the effective interaction between the electrons after integrating out the phonon mode has the form of $\zeta_{1}(\mathbf{k})$ as in our Eq. (1). While the existence of a nematic phase still needs further investigation, it is tempting to speculate that the non-Fermi-liquid behaviors in these systems can be partially attributed to the closeness of the nematic-isotropic transition, where the Fermi surface becomes soft. We note that our calculations are based on perturbation theory, ${ }^{32}$ and a detailed understanding of the fermionic properties near the QCP requires nonperturbative treatments of the theory, such as high-dimensional bosonization. ${ }^{24,33}$ However, it has been shown in Ref. 24 that perturbation theory does capture some of the essential physics.

In summary, we have studied the fluctuation effects of the diagonal electronic nematic order in the square lattice using a model Hamiltonian with a quadrupolar density-density interaction. We found that the collective mode associated with the diagonal nematic fluctuations has a strong anisotropy with diffusive peaks around the Fermi surface except along the $(0, \pm \pi)$ and $( \pm \pi, 0)$ directions. Close to the QCP, this mode becomes critical and strongly influences the decay rate of the quasiparticle; the imaginary part of the self-energy shows a non-Fermi-liquid behavior. We found that the decay rate at

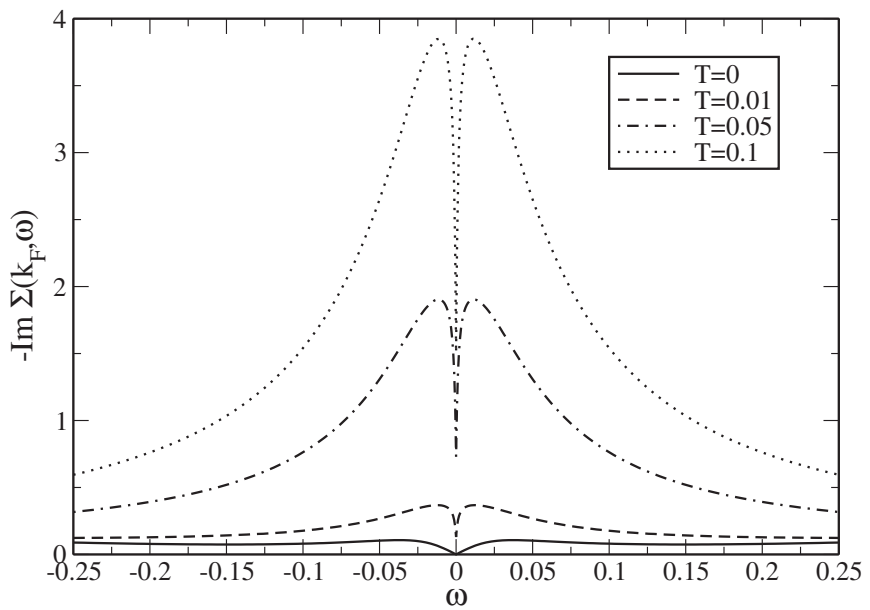

FIG. 3. Calculated frequency dependence of the quasiparticle self-energy at the Fermi surface along the $(\pi, \pi)$ direction at different temperatures with $\mu=-0.19$. The self-energy shows non-Fermiliquid behavior at small $\omega$ for all temperatures and scales as $\sqrt{T / \ln T}$ at $\omega=0$. All quantities are in units of $2 t$.

the Fermi surface is highly anisotropic and reaches a maximum at the QCP. It will be interesting to explore the effects of the collective mode on charge and heat transport, Raman spectroscopy, and the electron-phonon interaction near the transition.

\section{ACKNOWLEDGMENTS}

We thank T. P. Devereaux and N. Nagaosa for valuable discussions and J. Hopkinson for critical reading of the paper. We also thank KIAS for the hospitality where part of the work was carried out. This work was supported by NSC of Taiwan under Grant No. NSC-94-2112-M-002-047 and by the National Center for Theoretical Sciences (Y.-J.K.), NSERC of Canada, Canada Research Chair, Canadian Institute for Advanced Research, and Alfred P. Sloan Research Foundation (H.-Y.K.).
*Electronic address: yjkao@phys.ntu.edu.tw

†Electronic address: hykee@physics.utoronto.ca

${ }^{1}$ H. Doh, N. Friedman, and H.-Y. Kee, Phys. Rev. B 73, 125117 (2006).

${ }^{2}$ J. Tranquada, B. J. Sternlieb, J. D. Axe, Y. Nakamura, and S. Uchida, Nature (London) 375, 561 (1995).

${ }^{3}$ M. I. Salkola, V. J. Emery, and S. A. Kivelson, Phys. Rev. Lett. 77, 155 (1996).

${ }^{4}$ J. Zaanen and O. Gunnarsson, Phys. Rev. B 40, 7391 (1989).

${ }^{5}$ S. Mori, C. H. Chen, and S. W. Cheong, Nature (London) 392, 473 (1998).

${ }^{6}$ S. A. Kivelson, E. Fradkin, and V. J. Emery, Nature (London) 393, 550 (1998).

${ }^{7}$ S. A. Kivelson, E. Fradkin, V. Oganesyan, I. P. Bindloss, J. M. Tranquada, A. Kapitulnik, and C. Howald, Rev. Mod. Phys. 75,
1201 (2003).

${ }^{8}$ I. J. Pomeranchuk, Sov. Phys. JETP 8, 361 (1958).

${ }^{9}$ H. Yamase and H. Kohno, J. Phys. Soc. Jpn. 69, 2151 (2000).

${ }^{10}$ C. J. Halboth and W. Metzner, Phys. Rev. Lett. 85, 5162 (2000).

${ }^{11}$ V. Hankevych and F. Wegner, Eur. Phys. J. B 31, 333 (2003).

${ }^{12}$ J. Nilsson and A. H. Castro Neto, Phys. Rev. B 72, 195104 (2005).

${ }^{13}$ M. P. Lilly, K. B. Cooper, J. P. Eisenstein, L. N. Pfeiffer, and K. W. West, Phys. Rev. Lett. 82, 394 (1999).

${ }^{14}$ V. Hinkov, S. Pailhes, P. Bourges, Y. Sidis, A. Ivanov, A. Kulakov, C. T. Lin, D. P. Chen, C. Bernhard, and B. Keimer, Nature (London) 430, 650 (2004).

${ }^{15}$ Y.-J. Kao and H.-Y. Kee, Phys. Rev. B 72, 024502 (2005).

${ }^{16}$ S. A. Grigera, P. Gegenwart, R. A. Borzi, F. Weickert, A. J. Schofield, R. S. Perry, T. Tayama, T. Sakakibara, Y. Maeno, A. 
G. Green et al., Science 306, 1154 (2004).

${ }^{17}$ S. A. Grigera, R. A. Borzi, A. P. Mackenzie, S. R. Julian, R. S. Perry, and Y. Maeno, Phys. Rev. B 67, 214427 (2003).

${ }^{18}$ H.-Y. Kee and Y. B. Kim, Phys. Rev. B 71, 184402 (2005).

${ }^{19}$ V. Oganesyan, S. A. Kivelson, and E. Fradkin, Phys. Rev. B 64, 195109 (2001).

${ }^{20}$ H.-Y. Kee, E. H. Kim, and C.-H. Chung, Phys. Rev. B 68, 245109 (2003).

${ }^{21}$ I. Khavkine, C.-H. Chung, V. Oganesyan, and H.-Y. Kee, Phys. Rev. B 70, 155110 (2004).

${ }^{22}$ H. Yamase and W. Metzner, Phys. Rev. B 73, 214517 (2006).

${ }^{23}$ L. Dell'Anna and W. Metzner, Phys. Rev. B 73, 045127 (2006).

${ }^{24}$ M. J. Lawler, D. G. Barci, V. Fernandez, E. Fradkin, and L. Oxman, Phys. Rev. B 73, 085101 (2006).

${ }^{25}$ T. Moriya, Spin Fluctuations in Itinerant Electron Magnetism (Springer-Verlag, Berlin, 1985).
${ }^{26}$ W. Metzner, D. Rohe, and S. Andergassen, Phys. Rev. Lett. 91, 066402 (2003).

${ }^{27}$ A. J. Millis, Phys. Rev. B 48, 7183 (1993).

${ }^{28}$ S. Sachdev, Quantum Phase Transitions (Cambridge University Press, Cambridge, 1999).

${ }^{29}$ R. A. Borzi, S. A. Grigera, J. Farrell, R. S. Perry, S. J. S. Lister, S. L. Lee, D. A. Tennant, Y. Maeno, and A. P. Mackenzie, Science 315, 214 (2007).

${ }^{30}$ C. Stock, W. J. L. Buyers, R. Liang, D. Peets, Z. Tun, D. Bonn, W. N. Hardy, and R. J. Birgeneau, Phys. Rev. B 69, 014502 (2004).

${ }^{31}$ T. P. Devereaux, T. Cuk, Z.-X. Shen, and N. Nagaosa, Phys. Rev. Lett. 93, 117004 (2004).

${ }^{32}$ A. V. Chubukov, Phys. Rev. B 71, 245123 (2005).

${ }^{33}$ M. J. Lawler and E. Fradkin, Phys. Rev. B 75, 033304 (2007). 